\title{
Polymer-coated vertical-cavity surface-emitting laser diode vapor sensor
}

Ansbæk, Thor; Nielsen, Claus Højgaard; Larsen, Niels Bent; Dohn, Søren; Boisen, Anja; Chung, II-Sug; Larsson, David; Yvind, Kresten

\section{Published in:}

Proceedings of SPIE - The International Society for Optical Engineering

Link to article, DOI:

$10.1117 / 12.841935$

Publication date:

2010

Document Version

Publisher's PDF, also known as Version of record

Link back to DTU Orbit

Citation $(A P A)$ :

Ansbæk, T., Nielsen, C. H., Larsen, N. B., Dohn, S., Boisen, A., Chung, I-S., Larsson, D., \& Yvind, K. (2010). Polymer-coated vertical-cavity surface-emitting laser diode vapor sensor. Proceedings of SPIE - The International Society for Optical Engineering, 7615, 76150A. https://doi.org/10.1117/12.841935

\section{General rights}

Copyright and moral rights for the publications made accessible in the public portal are retained by the authors and/or other copyright owners and it is a condition of accessing publications that users recognise and abide by the legal requirements associated with these rights.

- Users may download and print one copy of any publication from the public portal for the purpose of private study or research.

- You may not further distribute the material or use it for any profit-making activity or commercial gain

- You may freely distribute the URL identifying the publication in the public portal 


\title{
Polymer-coated vertical-cavity surface-emitting laser diode vapor sensor
}

\author{
Thor Ansbæk ${ }^{a}$, Claus H. Nielsen ${ }^{b}$, Niels B. Larsen ${ }^{b}$, Søren Dohn ${ }^{b}$, Anja Boisen ${ }^{b}$, Il-Sug \\ Chung $^{a}$, David Larsson ${ }^{a}$ and Kresten Yvind ${ }^{a}$ \\ ${ }^{a}$ DTU Fotonik, Ørsteds Plads, Bygning 343, 2800 Kgs. Lyngby, Denmark; \\ ${ }^{b}$ DTU Nanotech, Ørsteds Plads, Bygning 345E, 2800 Kgs. Lyngby, Denmark
}

\begin{abstract}
We report a new method for monitoring vapor concentration of volatile organic compounds using a vertical-cavity surface-emitting laser (VCSEL). The VCSEL is coated with a polymer thin film on the top distributed Bragg reflector (DBR). The analyte absorption is transduced to the electrical domain through modulation of the VCSEL output power as the polymer swell. We have investigated the responsivity of this technique experimentally using a plasma polymerized polystyrene coating and explain the results theoretically as a reflectance modulation of the top DBR.
\end{abstract}

Keywords: vertical-cavity surface-emitting laser, functionalization, sensor polymer, vapor sensor, volatile organic compound, gas sensing

\section{INTRODUCTION}

The vertical-cavity surface-emitting laser (VCSEL) has found numerous uses within position detection and gas absorption spectroscopy due to its low power consumption and low system cost. ${ }^{1-3}$ In such applications the good single-mode properties and low astigmatism make it possible to construct simple reflection-based systems. We have earlier shown modulation of the output power of a VCSEL using self-mixing interferometry with the external reflector being a movable cantilever. ${ }^{4}$ By coating the cantilever with a thin polymer, vapor absorption would cause swelling of the polymer and the associated strain translates into a cantilever bending. This is detected as change in the VCSEL output power. This sensor system require very precise mechanical interfacing between the VCSEL and cantilever which is a disadvantage in terms of stability. Here we present a new and simpler approach where the polymer is applied directly to the top distributed Bragg reflector (DBR). The polymer swelling cause a change in the optical thickness of the low index polymer that modulates the reflectance of the top facet. This changes the threshold condition of the Fabry-Pérot cavity and the light extraction of the top facet relative to the bottom facet. Here we show both theoretically and experimentally that this can be detected as a change in the top facet power output. Detection of the power output, to say the diode voltage or wavelength, makes for large relative responsivity which can be detected by simple electronic signal conditioning using e.g. low cost $\mathrm{Si}$ photodiodes. The analyte-polymer system chosen for investigation was that of acetone vapor sorption in polystyrene, but any polymer that changes its optical thickness upon exposure to the target vapor can be used. Here we present the first measurement of the acetone concentration in a measurement chamber with $25000 \mathrm{ppm}$ sensitivity. By monitoring the light-current characteristic, instead of the power at one bias current, we are able to resolve the change in both differential quantum efficiency and threshold current. We show that (monitoring the top coated facet) for small changes in the optical thickness the highest sensitivity is found close to the threshold current.

Further author information: (Send correspondence to T.A.)

T.A.: E-mail: tans@fotonik.dtu.dk, Telephone: +45 45253643 www.fotonik.dtu.dk/devices

Vertical-Cavity Surface-Emitting Lasers XIV, edited by James K. Guenter, Kent D. Choquette, Proc. of SPIE Vol. 7615, 76150A - (c) 2010 SPIE · CCC code: 0277-786X/10/\$18 · doi: 10.1117/12.841935 


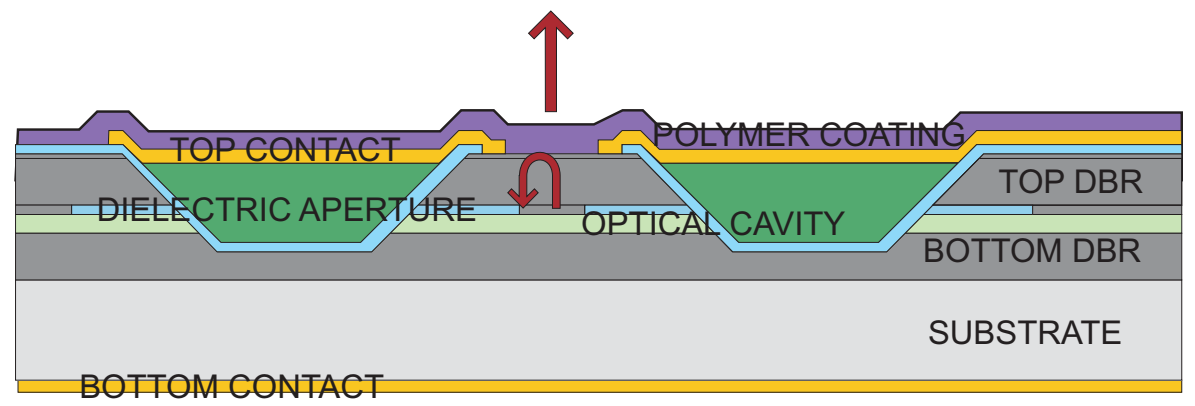

Figure 1. Schematic drawing of the device structure.

\section{THEORY}

\subsection{Laser diode model}

The optical thickness change of the polymer film modulates the mirror reflectance with a period of $\lambda_{0} / 2$, where $\lambda_{0}$ is the lasing wavelength. The change in mirror reflectance and transmittance alters the threshold condition of the laser cavity, thus changing the output power. Hence the output power is a function of the optical thickness, or the optical phase $\phi$, of the sensor polymer coating of the top DBR. In steady-state where stimulated emission is dominant the optical power output can be written as

$$
P_{t}(\phi)=\eta_{d, t}(\phi) \frac{h \nu}{q}\left(I-I_{t h}(\phi)\right) \quad I>I_{t h}
$$

where $h$ is Planck's constant, $\nu$ the lasing frequency, $q$ the electron charge, $\eta_{d, t}$ the differential quantum efficiency for the light output of the top mirror, $I$ the bias current, $I_{t h}$ the threshold current and $\phi$ is the optical phase of the sensor polymer

$$
\phi=\frac{2 \pi}{\lambda_{0}} n_{p} d_{p}
$$

with $\lambda_{0}$ being the wavelength of the emitted light, $n_{p}$ and $d_{p}$ the refractive index and thickness of the polymer, respectively. ${ }^{5}$ The index $t$ denote that we look at the light detected at the top mirror. To the first order we neglect the phase-dependence of the lasing wavelength and thus only $\eta_{d, t}$ and $I_{t h}$ depends on the optical phase $\phi$ as indicated in Eq. 1. Both variables relate to the mirror loss $\alpha_{m, t}$ by the threshold condition $\Gamma g_{t h}(\phi)=$ $\left\langle\alpha_{i}\right\rangle+\alpha_{m, t}(\phi)$, the leftside being the modal gain and the rightside the total loss. The differential quantum efficiency in terms of the top mirror loss is

$$
\eta_{d t}(\phi)=\eta_{i} F_{t}(\phi) \frac{\alpha_{m, t}(\phi)}{\left\langle\alpha_{i}\right\rangle+\alpha_{m, t}(\phi)},
$$

where $\eta_{i}$ is the internal quantum efficiency, $F_{t}$ the fractional output of the top mirror and $\left\langle\alpha_{i}\right\rangle$ the cavity loss. The fractional output corrects for the fact that the laser cavity is assymmetrical in terms of the reflectance of the top and bottom mirrors. Thus the ratio of interest is the output power from the top mirror from which the emitted light is detected to the total power coupled out of the laser. The fractional output power of the mirror $F_{t}$ is given in terms of the reflectance $R_{t / b}$ and transmittance $T_{t / b}$ of the top and bottom mirror respectively

$$
F_{t}(\phi)=\frac{T_{t}(\phi)}{\left(1-R_{t}(\phi)\right)+\sqrt{\frac{R_{t}(\phi)}{R_{b}}}\left(1-R_{b}\right)} .
$$

Assuming a logarithmic gain-current relation the threshold carrier density depends exponentially on the mirror loss as

$$
N_{t h}(\phi)=N_{t r} \exp \left\{\frac{\left\langle\alpha_{i}\right\rangle+\alpha_{m, t}(\phi)}{N_{w} \Gamma_{1} g_{0}}\right\},
$$


where $N_{t r}$ is the transparency carrier density, $\Gamma_{1}$ the single-well confinement factor, $N_{w}$ the number of quantum wells and $g_{0}$ the gain coefficient. Thus the threshold current also depends exponentially on the mirror loss. Using a polynomial expansion of the recombination rate in terms of the parameters $A, B$ and $C$ the threshold current can be written as

$$
I_{t h}(\phi)=\frac{q N_{w} V_{1}}{\eta_{i}}\left[A N_{t h}(\phi)+B N_{t h}(\phi)^{2}+C N_{t h}(\phi)^{3}\right] .
$$

where $V_{1}$ is the single-well volume $V_{1}=d_{1} \pi w^{2}, d_{1}$ being the thickness of a single well and $w$ the radius of the current aperture. Assuming that non-radiative and Auger recombination are negligible compared to the spontaneous emission we can set $A=0$ and $C=0$, respectively. The mirror loss is by definition

$$
\alpha_{m, t}(\phi) \equiv \frac{1}{L} \ln \left(\frac{1}{R(\phi)}\right)
$$

where $R=\left|r_{t} r_{b}\right|$ is the mirror reflectance given by the top $r_{t}$ and bottom $r_{b}$ mirror field reflection coefficients. The cavity length is taken to be $L=\frac{\lambda_{0}}{n_{s}}$, where $n_{s}$ is the refractive index of the cavity. Hence we take the change in the penetration depth to be negligible and constant with $\phi$. The mirror reflectance is found using the transfer-matrix method. ${ }^{6}$ For the ideal loss-less mirror the reflectance top mirror reflectance $R_{t}=r_{t} r_{t}^{*}$ is found to be

$$
\begin{aligned}
R(\phi) & =\frac{\left(n_{s}\left(\frac{n_{L}}{n_{H}}\right)^{2 N}-n_{0}\right)^{2} \cos ^{2}(\phi)+\left(\frac{n_{s} n_{0}}{n_{p}}\left(\frac{n_{L}}{n_{H}}\right)^{2 N}-n_{p}\right)^{2} \sin ^{2}(\phi)}{\left(n_{s}\left(\frac{n_{L}}{n_{H}}\right)^{2 N}+n_{0}\right)^{2} \cos ^{2}(\phi)+\left(\frac{n_{s} n_{0}}{n_{p}}\left(\frac{n_{L}}{n_{H}}\right)^{2 N}+n_{p}\right)^{2} \sin ^{2}(\phi)} \\
& =\frac{n_{A}+\left(n_{B}-n_{A}\right) \sin ^{2}(\phi)}{n_{C}+\left(n_{D}-n_{C}\right) \sin ^{2}(\phi)}
\end{aligned}
$$

where $N$ is the number of periods. The prefactors $n_{A}, n_{B}, n_{C}, n_{D}$ are defined in terms of the refractive index of the top DBR high $n_{H}$ and low $n_{L}$ index layers, the cavity $n_{s}$, the media surrounding the VCSEL $n_{0}$ and the polymer $n_{p}$. The two prefactors $n_{B}$ and $n_{D}$ are taken to be constant, hence neglecting the small variation due to changes in $n_{p}$. Note that $n_{0}$ is taken to be the refractive index of free space and thus is a constant. In a volatile organic compound (VOC) detection setup where high concentrations are encountered $n_{0}$ will also depend on the vapor concentration. The power output of the VCSEL versus the polymer coating optical phase can be simulated with the model as given by Eqs. (1) to (2). This has been done using the parameters seen in Tab. 1, where standard values for $850 \mathrm{~nm}$ GaAs/AlGaAs quantum well (QW) VCSELs are used. Fig. 2 shows the

Table 1. Standard laser parameters of $850 \mathrm{~nm}$ GaAs/AlGaAs quantum well (QW) VCSELs. ${ }^{5}$

\begin{tabular}{|l|c|l|}
\hline Parameter & Value & Unit \\
\hline \hline$\lambda_{0}$ & 850 & $\mathrm{~nm}$ \\
\hline$\Gamma g_{0}$ & 52.5 & $\mathrm{~cm}^{-1}$ \\
\hline$N_{t r}$ & $2.6 \times 10^{18}$ & $\mathrm{~cm}^{-3}$ \\
\hline$N_{w}$ & 3 & \\
\hline$d_{1}$ & 8 & $\mathrm{~nm}$ \\
\hline$w$ & 2 & $\mu \mathrm{m}$ \\
\hline$\left\langle\alpha_{i}\right\rangle$ & 20 & $\mathrm{~cm}^{-1}$ \\
\hline$\eta_{i}$ & 0.8 & \\
\hline$B$ & $0.8 \times 10^{-10}$ & $\mathrm{~cm}^{3} \mathrm{~s}^{-1}$ \\
\hline$N_{\text {top }} / N_{\text {bottom }}$ & $26 / 30$ & \\
\hline$I_{\text {th }}$ & $1.05 I_{\text {th }}^{\max }$ & $\mathrm{mA}$ \\
\hline \multicolumn{2}{|l}{} \\
\multicolumn{2}{|l}{} \\
\end{tabular}

laser diode normalized threshold current $I_{t h} / I_{t h, 0}$ (solid line) and the differential quantum efficiency $\eta_{d, t}$ (dashed 
line) as a function of the polymer film optical phase. The threshold current has been normalized to show the dependence on $\phi$ only since we are not interested in modelling the absolute threshold current. The threshold carrier density depends exponentially on the total loss, that is the sum of all optical losses, and thus is a product of several parts where only the exponential to the mirror loss is important to see the dependence on $\phi$. Hence to simplify the analysis we neglect diffraction losses from the dielectric aperture as well as other recombinations mechanisms than spontaneous emission. The diffraction loss is strongly dependent on the aperture radius and the refractive index discontinuities for single-mode aperture sizes and thus strongly dependent on the exact structure of the VCSEL. ${ }^{7,8}$ Both the threshold current and the the differential quantum efficiency increase with $\phi$ until $\phi=\pi / 2$. This is in accordance with the expected results as the gain required for lasing is expected to increase as the mirror reflectance decreases. The rise in differential quantum efficiency is also expected as more light is extracted from the top mirror relative to the bottom mirror as the top mirror reflectance decreases. A sinusoidal dependence is seen which is expected from Eq. 8 and hence the $\frac{\lambda_{0}}{2}$ periodicity derives from Eq. (2). Fig. 3

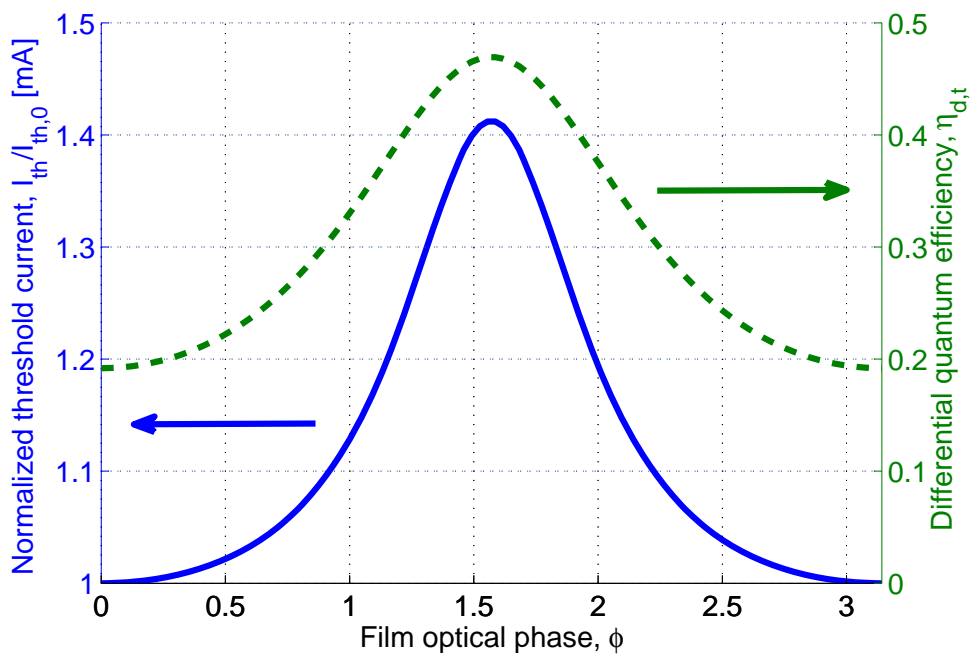

Figure 2. Theoretical calculation of the threshold current $I_{t h}$ (dashed line, left axis) and different quantum efficiency $\eta_{d, t}$ (solid line, right axis).

shows the normalized laser diode output power $P_{t} / P_{t, 0}$ as a function of the polymer film optical phase where $P_{t, 0}=P_{t}(\phi=0)$. The output power is plotted for two different bias conditions: very close to the threshold current $\left(I=1.05 I_{t h}^{\max }\right)$ and far above the threshold current $\left(I=3 I_{t h}^{\max }\right)$, where $I_{t h}^{\max }$ is the maximum threshold current $I_{t h}^{\max }=I_{t h}(\phi=\pi / 2)$. From a sensitivity analysis of Eq. (1) we find that

$$
\mathcal{S}_{P_{t}}^{\phi}=\frac{\phi}{P_{t}} \frac{\delta P_{t}}{\delta \phi}=\frac{\phi}{\eta_{d, t}} \frac{\partial \eta_{d, t}}{\partial \phi}-\frac{\phi}{I-I_{t h}} \frac{\partial I_{t h}}{\partial \phi}
$$

where for $I \gg I_{t h}$ the power output is mainly dependent on the change in $\eta_{d, t}$ while for $I \rightarrow I_{t h}$ the change in threshold current is dominant. This is also seen from the simulation which for large bias current show a maximum in power output at $\pi / 2$ for both the differential quantum efficiency and the power output. In the case of a bias current close to threshold the power output show a minimum at $\pi / 2$ due to the maximum in threshold current. From Eq. (9) it is seen that the two contributions cancel out. Hence either a bias condition close to threshold or far above should be used to operate away from the point of zero sensitivity which is approximately at

$$
I_{\mathcal{S}_{P_{t}}^{\phi}=0}=\Delta\left(\eta_{d, t} I_{t h}\right) / \Delta \eta_{d, t}
$$

where $\Delta\left(\eta_{d, t} I_{t h}\right)$ is the change in the product of the differential quantum efficiency and the threshold current. ${ }^{4}$ We note that this zero sensitivity bias point is only present for a design where light emitted from the coated facet is detected. In case we look at the light output from the opposite facet the two contribution in Eq. 9 add 
up making for a larger responsivity. ${ }^{9}$ From Fig. 3 it is also seen that the polymer coating thickness choosen to give either high sensitivity or dynamic range depends on the bias condition.

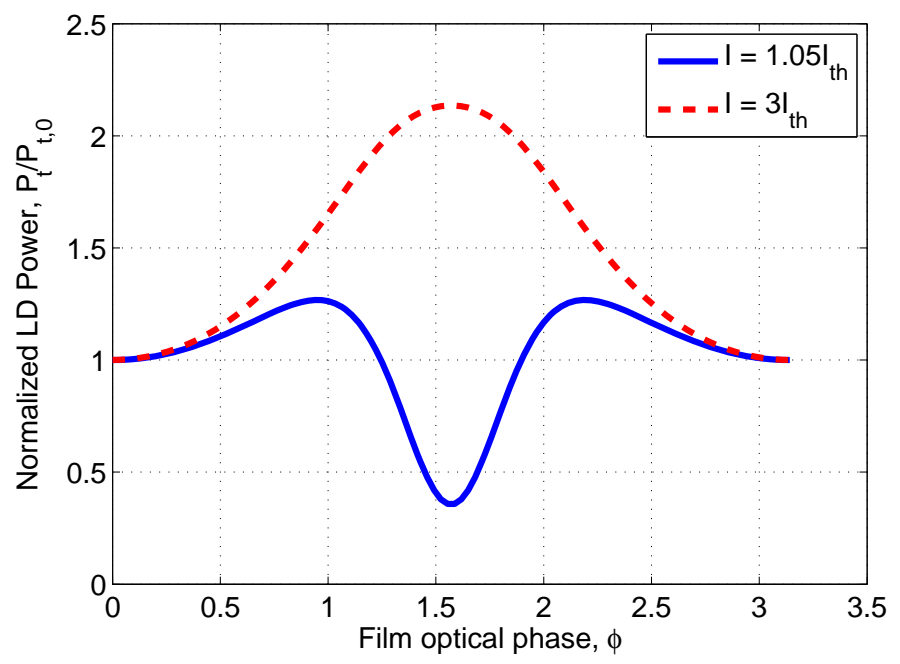

Figure 3. Theoretical calculation of the output power $P$ for two different bias conditions; close to threshold (solid line) and far above threshold (dashed line).

\section{EXPERIMENT}

An experiment was designed to test whether as expected from theory the VCSEL power output would depend on the coating thickness. The application in mind is vapor sensing and for testing this a polystyrene coating was used in a acetone flow setup. Single-mode $850 \mathrm{~nm}$ VCSEL diodes (Finisar ${ }^{\circledR}$ HFE4093-332) were used for the acetone response experiment: (1) one used as reference, (2) the other coated with polystyrene (PS). The PS was deposited on the top p-doped DBR.

\subsection{Polymer coating}

The deposition method used was plasma polymerization, which is a solvent-free technique. This ensures that the polymer is not already containing the solvent we want to detect. The polymerization was done using a plasma chamber with a styrene monomer source at constant pressure. The plasma polymerization was done at low power and frequency to preserve the monomer functional vinyl groups responsible for polymerization. The polystyrene film deposited on a silicon piece placed in the plasma chamber during deposition was measured by spectroscopic ellipsometry to have a thickness of $79 \pm 3 \mathrm{~nm}$ and a refractive index of $1.614 \pm 0.002 @ 850 \mathrm{~nm}$. From the optical thickness $127.5 \mathrm{~nm}$ we estimate $\phi=0.94$.

\subsection{Flow measurement}

The light-current-voltage (LIV) characteristics of each VCSEL was measured before and after coating. For the acetone response measurement the VCSELs were mounted on a custom aluminum holder and connected to two laser drivers. The laser drivers were used to sweep a forward bias current from 0.5 to $2 \mathrm{~mA}$ and measuring the voltage drop across the VCSELs. The output power from the VCSELs was measured with two silicon photodiodes (PD), both connected to a current meter. The responsivity of the photodiodes used is $0.56 \mathrm{~A} / \mathrm{W}$ at a wavelength of $850 \mathrm{~nm}$. The current meter has a 13 bit resolution $0.2 \mu \mathrm{A}$, thus the resolution of the detected power with the range set to $\pm 2 \mathrm{~mA}$ should be $P_{\text {resolution }}=0.9 \mu \mathrm{W}$. The aluminum holder was placed in a vacuum chamber with electrical connections. The chamber has three inlet lines: (1) one $\mathrm{N}_{2}$ source line going through an acetone bubbler, (2) one $\mathrm{N}_{2}$ dilution line and (3) one $\mathrm{N}_{2}$ purge line. All lines are controlled with a magnetic valve (MV) and the bubbler and purge line has a mass flow controller (MFC) to control the $\mathrm{N}_{2}$ flow. The dilution line flow 


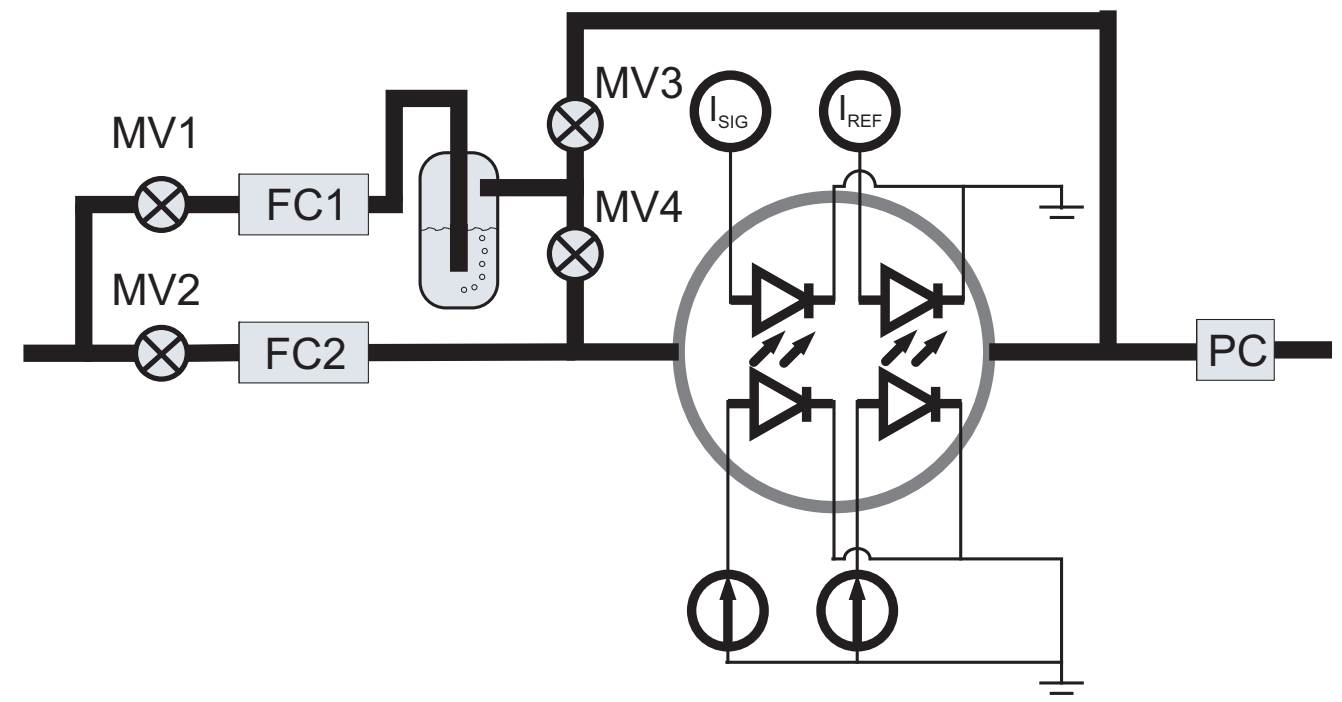

Figure 4. Schematic drawing of the experimental setup used for the experiments. An nitrogen source is split into two lines controlled by a mass flow controller (MFC) and a third line for purge the chamber. Each controlled by a magnetic valve (MV). The outlet of the bubbler is split into a line going to the chamber and a line bypassing the chamber, both controlled by a MV. The outlet of the chamber is controlled by a pressure controller (PC) and a magnetic valve in series with a MFC.

(FC1) was controlled such as to keep the total flow $Q_{\text {tot }}=50 \mathrm{sccm}$. The bubbler further has a line bypassing the chamber, which is controlled by MV3 and MV4 such that the flow can be directed either to or pass the chamber. The outlet of the chamber is connected to a pump and controlled by a pressure controller (PC), keeping the chamber pressure constant at 600 Torr. The volume $V$ of the chamber is $1800 \mathrm{~cm}^{2}$ and with a outlet flow equal to $Q_{\text {tot }}$ this gives a time constant $\tau$ of 36 minutes. The relative acetone concentration in the chamber during filling and purging can be estimated to be

$$
C_{\text {acetone }}(t)=C_{0} \times\left\{\begin{array}{rl}
\exp \left\{-\frac{t}{\tau}\right\} & \text { purging } \\
1-\exp \left\{-\frac{t}{\tau}\right\} & \text { filling }
\end{array},\right.
$$

where $C_{0}$ is the relative acetone concentration at the end of the filling or purging period, respectively. For the initial filling the relative acetone concentration is

$$
C_{0}=\frac{p_{\mathrm{svp}}^{\text {acetone }}}{p_{\text {chamber }}-p_{\mathrm{svp}}^{\text {acetone }}} \epsilon \frac{Q_{\text {bubbler }}}{Q_{\text {tot }}},
$$

assuming tubing of infinite conductance, constant flow rates $\left(Q_{\text {bubbler }}, Q_{\text {tot }}\right)$ and an initial relative acetone concentration of zero $\left(C_{\text {acetone }}(t)=0\right)$. The saturated vapor pressure $p_{\text {acetone }}^{\text {svp }}$ of acetone is 175 Torr and thus the condition for Eq. $12, p_{\text {chamber }}>3 p_{\text {svp }}$ is true. ${ }^{10}$ The chamber was first pumped to 37.5 Torr and purged with nitrogen to reach a chamber pressure of 600 Torr. This cycle was repeated 10 times to remove water vapor. The chamber now considered a nitrogen atmosphere was then filled with acetone setting $Q_{\text {bubbler }}=20$ sccm for 180 min, after which the chamber was purged with a total flow of $Q_{t o t}=50 \mathrm{sccm}$ for 180 min. The sequence was repeated thrice. During measurement the chamber temperature was measured to vary by $\pm 0.2^{\circ}$.

\section{RESULTS \& DISCUSSION}

The treshold current, $I_{t h}$, before and after the polystyrene coating was found to be $0.9 \mathrm{~mA}$ and $1.14 \mathrm{~mA}$, respectively. Fig. 5 shows the light-current characteristic of the laser diode before (dashed line) and after coating (solid line). The threshold current $I_{t h}$ and differential quantum efficiency $\eta_{d, t}$ was extracted using a linear fit of Eq. 1 in the current interval $I \in[1.25 ; 1.75]$. From this it is found that both $I_{t h}$ and $\eta_{d, t}$ increased after coating with polystyrene. The agrees with the theoretical results, see Fig. ??. 


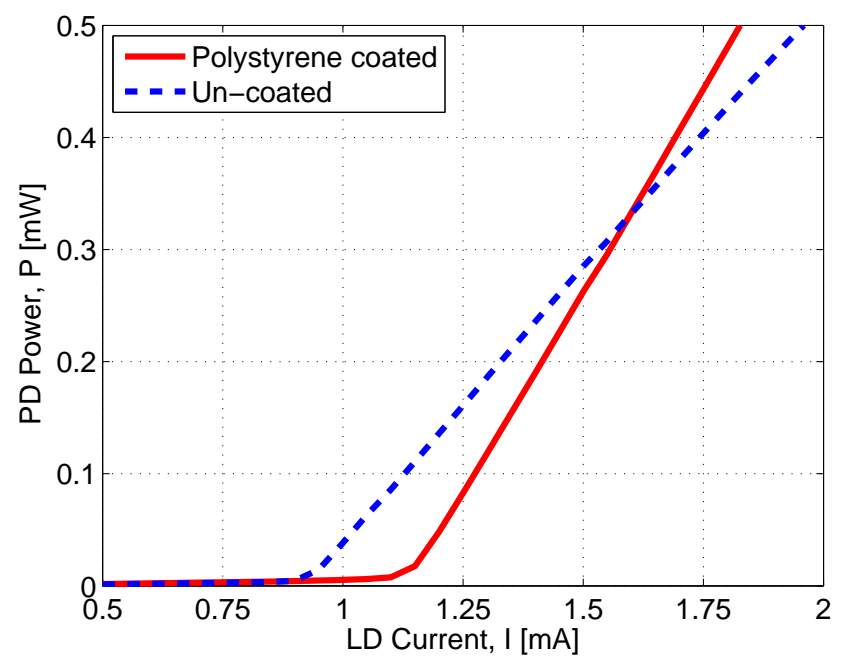

Figure 5. Graph showing the light-current characteristic of the laser diode before (dashed line) and after (solid line) coating the top DBR with polystyrene. The differential quantum effiency $\eta_{d, t}$ before and after coating was 0.334 and 0.496, respectively. The threshold current $I_{t h}$ before and after coating was $0.92 \mathrm{~mA}$ and $1.14 \mathrm{~mA}$, respectivly. From these values the bias point of zero sensitivity can be estimate from Eq. 10 to be $1.60 \mathrm{~mA}$ as is also seen in the graph.

Fig. 6 shows the detected power at the photodiode versus time of the second fill/purge sequence. The laser diode bias current is $I=1.15 \mathrm{~mA}$, which is just above the threshold current at time zero. Both the laser response (dashed line) and the calculated acetone concentration (solid line) are shown. The acetone concetration was calculated from Eqs. 11 and 12. The VCSEL power is seen to be a directly correlated to the calculated acetone concentration. The detected power has been corrected for drift to be able to be able compare it to the acetone concentration. This was done using a linear fit to the power before and after the measurement sequence as base level. The inset show the power detected from the un-coated reference laser diode. The power drift is seen to be linear with time, which thus justify the approach. The signal drift was comparable to the signal magnitude and found to be larger for the coated than the un-coated diode. This could indicate that the drift is connected to both the polymer and the laser diode. The power output trace the acetone concentration during purging, but not during the fill period. That the VCSEL power does not respond to the acetone concentration on the time scale of the experiment indicate that the polymer swelling is limited by diffusion on a timescale comparable to the filling time constant of the chamber. The laser diode power output detected at the photodiode is seen to vary from $60 \mu \mathrm{W}$ at an acetone concentration below $1 \%$ to $82 \mu \mathrm{W}$ at an acetone concentration of $16 \%$. Hence the responsivity can be approximated to be $0.1 \mathrm{nW} / \mathrm{ppm}$.

Fig. 6 shows the detected power at a specific laser diode bias current only. The curve was extracted from a full light-current measurement of the laser diode sampled with a $33 \mathrm{mHz}$ repetition rate. The light-current characteristic at the rising period of the curve is seen in Fig. 7. The current is stepped with an interval of $50 \mu \mathrm{A}$. The differential quantum efficiency and the threshold current was extracted using a linear fit in the current interval $I \in[1.15 ; 1.3]$. Fig. 7 shows that both the threshold current and differential quantum efficiency decreases with time. From theory we would expect this to be the case when the optical thickness is larger than $\lambda_{0} / 4$. This is not in agreement with the measured optical thickness and is attributed to the coating thickness being different on the VCSEL.

The rationale for monitoring the power at a bias current of $I=1.15 \mathrm{~mA}$ is that the response is at a maximum. ${ }^{3}$ Using the values found for the change in threshold current and differential quantum efficiency from Fig. 7 and Eq. (10) it is found that the zero sensitivity point is at $I_{\mathcal{S}_{P_{t}}^{\phi}=0}=3.35 \mathrm{~mA}$, which is above the current range for the amperemeter as well as the temperature roll-off. Hence the sensitivity goes towards zero for increasing bias currents. Fig. 8 shows the measured relative response versus the bias current. The graph shows that the relative response is largest close to the threshold current $I_{t h}$ and falls off above the threshold current. Below the 


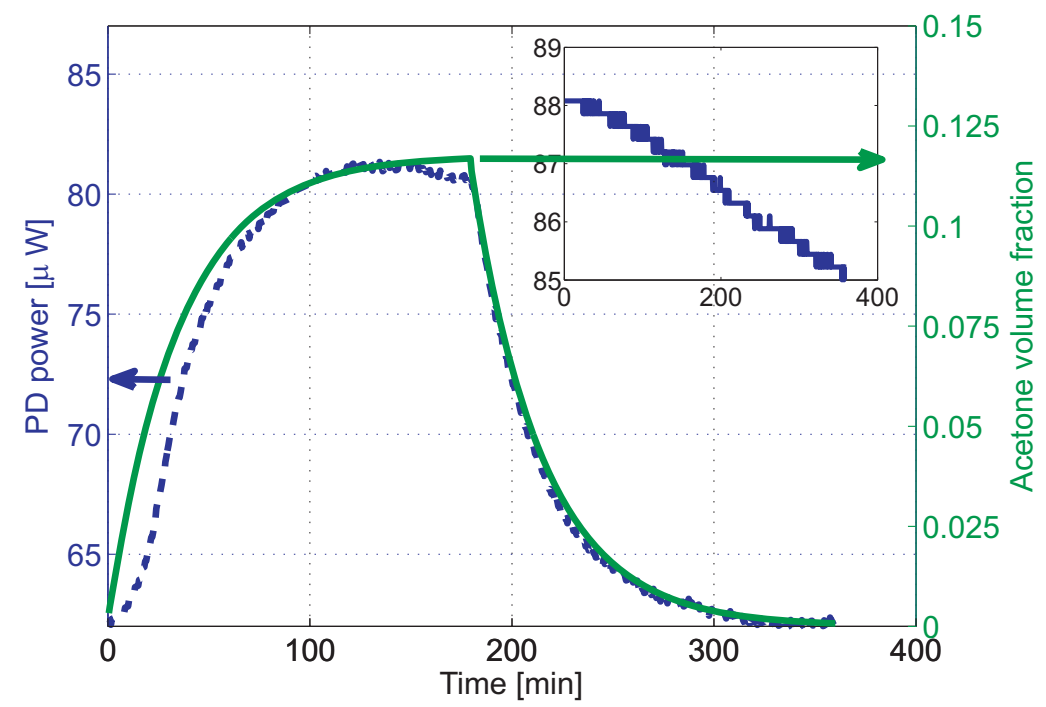

Figure 6. Graph showing the response of the sensor to acetone for a 180 minute fill period and 180 minute purge period. The detected power at the photodiode (dashed line, left axis) is plotted against the calculated acetone concentration (solid line, right axis). The response is shown for a laser diode bias of $I=1.15 \mathrm{~mA}$. The inset show the detected power of the reference laser diode.

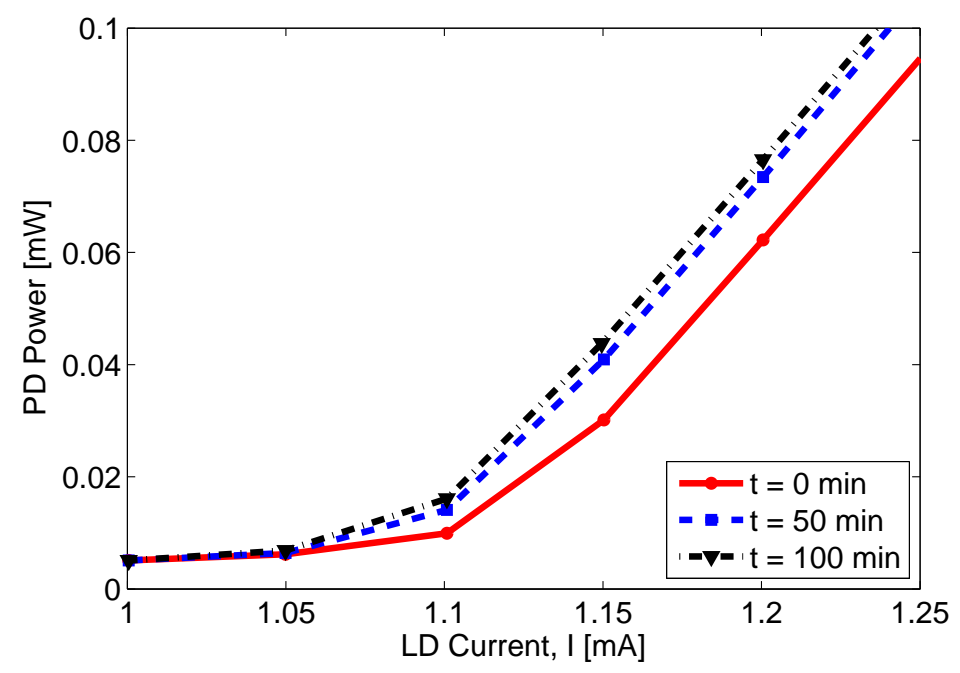

Figure 7. Graph showing the light-current curve for three different times $t=0, t=50$ min and $t=100$ min relative to the opening of the valve to the acetone bubbler. The differential quantum efficiency $\eta_{d, t}$ with increasing time $t$ was 0.449 , 0.448 and 0.445 , respectively. The threshold current $I_{t h}$ was $1.10,1.09$ and 1.08 , respectively.

threshold current the relative response is zero since the signal is small (spontaneous emission) and not sensitive to the variation in mirror reflection. Also shown in Fig. 8 is the signal-to-noise ratio $\mathrm{SNR}=\Delta P_{t} / P_{\text {resolution }}$ as a function of laser diode bias current. The figure of merit is the product of the relative change and the signal to noise ratio which has its maximum at $I=1.15 \mathrm{~mA}$. The acetone sensitivty was estimated from the noise floor to be around $25000 \mathrm{ppm}$. To achieve a better sensitivity the polymer thickness must be precisely controlled such that the sensitivity is maximum at the bias operating point. The power sensitivity can be increased by using amplified photodiodes or the lock-in technique, but signal drift must be minimized. Sources of signal drift from the laser diode are the temperature dependence of the gain and recombination rate. This dealt with by using a thermoelectric cooler (TEC) and a current-control feedback loop using power monitoring of an identical 


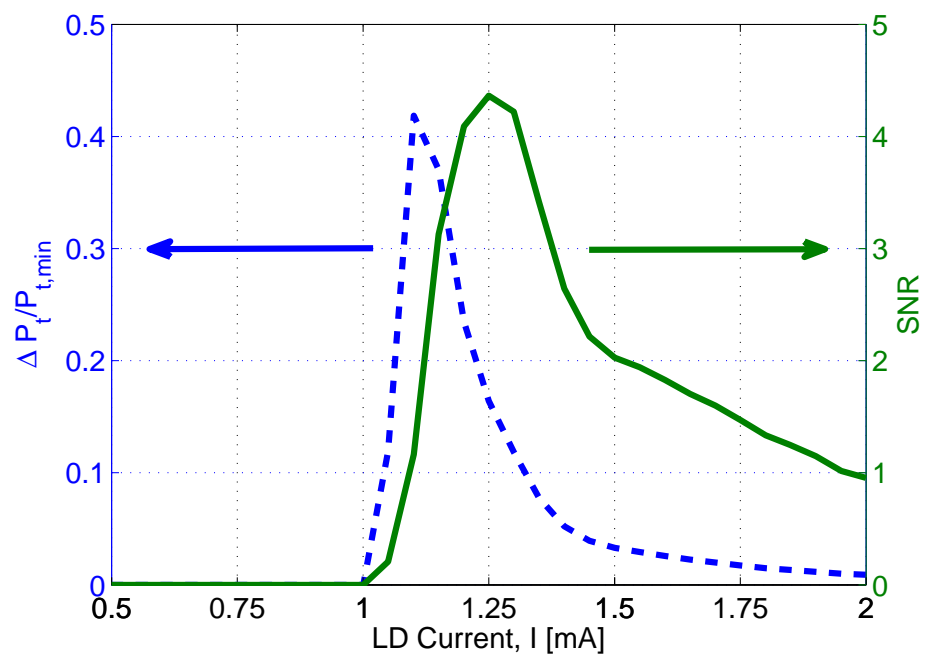

Figure 8. Graph showing the experimental relative acetone response $\frac{\Delta P_{t}}{P_{t, \min }}$ versus the laser diode bias current $I$. The relative response is seen to be largest at a bias current of $I=1.15 \mathrm{~mA}$, which is close to the threshold current $I_{t h}$ and the maximum in signal-to-noise ratio $\mathrm{SNR}=\frac{\Delta P_{t}}{P_{\text {resolution }}}$.

un-coated laser diode. Another drift source is the temperature and acetone partial pressure dependency of the diffusion constant of the polymer. The can be dealt with by changing the measurement setup, but will be present in a real-life measurement.

\section{CONCLUSION}

We have demonstrated a new vapor sensing technique based on a polymer coated VCSEL diode. Using a simple flow model we conclude that the sensor can monitor the acetone vapor concentration in the measurement chamber. Using the steady-state solution of the laser rate equations and the transfer-matrix method to calculate the mirror reflectance we show theoretically the dependence of the power output on the polymer thickness. The optical phase shows a periodicity of $\lambda_{0} / 2$, where $\lambda_{0}$ is the lasing wavelength. From the light-current characteristic of the VCSEL we deduce that the threshold current and differential quantum efficiency decrease with increasing acetone concentration for the given sensor design and bias current. The sensitivity is shown to be at a maximum around the threshold current. This vapor sensing technique has the advantages of enabling quantative assay measurements, being mechanically stable and versatile in terms of coating used.

\section{ACKNOWLEDGMENTS}

The work was supported by the Danish strategic research council through the Nanonose project.

\section{REFERENCES}

[1] Allen, M., "Diode laser absorption sensors for gas-dynamic and combustion flows," Measurement Science and Technology 9, 545-562 (1998).

[2] de Groot, P., Gallatin, G., and Macomber, S., "Ranging and velocimetry signal generation in a backscattermodulated laser diode," Applied Optics 27(21), 4475-4480 (1988).

[3] Wang, W., Grattan, K., Palmer, A., and Boyle, W., "Self-mixing interference inside a single-mode diode laser for optical sensing applications," Lightwave Technology, Journal of 12(9), 1577-1587 (1994).

[4] Larsson, D., Greve, A., Hvam, J., Boisen, A., and Yvind, K., "Self-mixing interferometry in vertical-cavity surface-emitting lasers for nanomechanical cantilever sensing," App. Phys. Lett., 94, 091103-3 (2009). 
[5] Coldren, L. A. and Corzine, S. W., [Diode Lasers and Photonic Integrated Circuits], John-Wiley and Sons, New York, first ed. (1995).

[6] Pedrotti, F. L. and Pedrotti, L. S., [Introduction to Optics], Printice-Hall, New-Jersey, second ed. (1959).

[7] Choquette, K. D., Chow, W. W., Hadley, G. R., Hou, H. Q., and Geib, K. M., "Scalability of small-aperture selectively oxidized vertical cavity lasers," App. Phys. Lett., 70, 823-825 (1997).

[8] Hegblom, E. R., Babic, D. I., Thibeault, B. J., and Coldren, L. A., "Scattering losses from dielectric apertures in vertical-cavity lasers," IEEE J. Sel. Top. Quantum Electron. 70, 823-825 (1997).

[9] Larsson, D., Yvind, K., Chung, I.-S., and Hvam, J., "Optimization of vcsel for self-mixing sensing," IEEE Photonics Technol. Lett., . To be published.

[10] Breiland, W. G., Coltrin, M. E., Creighton, J. R., Hou, H. Q., Moffat, H. K., and Tsao, J. Y., "Organometallic vapor phase epitaxy (omvpe)," Mater Sci Eng R24, 241-274 (1999). 